\title{
Population genomic analysis provides strong evidence of the past success and future strategies of South China tiger breeding
}

\section{Chen Wang}

Guangzhou Zoo \& Guangzhou Wildlife Research Center

\section{Dong-Dong Wu}

State Key Laboratory of Genetic Resources and Evolution, Kunming Institute of Zoology, Chinese Academy of Sciences, Kunming, Yunnan, 650223 https://orcid.org/0000-0001-7101-7297

\section{Yao-Hua Yuan}

Shanghai Zoo

\section{Meng-Cheng Yao}

Kunming Institute of Zoology, Chinese Academy of Sciences

Jian-Lin Han

https://orcid.org/0000-0002-1527-3963

\section{Ya-Jiang Wu}

Guangzhou Zoo \& Guangzhou Wildlife Research Center

\section{Fen Shan}

Guangzhou Zoo \& Guangzhou Wildlife Research Center

Wan-Ping Li

Guangzhou Zoo \& Guangzhou Wildlife Research Center Jun-Qiong Zhai

Guangzhou Zoo \& Guangzhou Wildlife Research Center

\section{Mian Huang}

Guangzhou Zoo \& Guangzhou Wildlife Research Center

\section{Shi-Ming Peng}

Guangzhou Zoo \& Guangzhou Wildlife Research Center

\section{Qin-Hui Cai}

Guangzhou Zoo \& Guangzhou Wildlife Research Center

\section{Jian-Yi Yu}

Shanghai Zoo

Qun-Xiu Liu

Shanghai Zoo

\section{Zhao-Yang Liu}

Wangcheng Park 


\section{Lin-Xiang Li}

Suzhou Shangfangshan Forest Zoo

\section{Ming-Sheng Teng}

Chongqing Zoo

\section{Wei Huang}

Nanchang Zoo

Jun-Ying Zhou

Chinese Association of Zoological Gardens

Chi Zhang

BGI genomics

Wu Chen ( $\nabla$ guangzhouchenwu@sina.com )

Guangzhou Zoo

\section{Xiao-Long Tu}

Kunming Institute of Zoology, Chinese Academy of Sciences

\section{Article}

Keywords:

Posted Date: December 21st, 2021

DOI: https://doi.org/10.21203/rs.3.rs-1157812/v1

License: (c) (1) This work is licensed under a Creative Commons Attribution 4.0 International License. Read Full License 


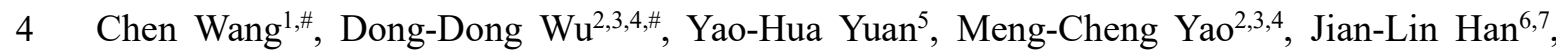

5 Ya-Jiang $\mathrm{Wu}^{1}$, Fen Shan ${ }^{1}$, Wan-Ping $\mathrm{Li}^{1}$, Jun-Qiong Zhai ${ }^{1}$, Mian Huang ${ }^{1}$, Shi-Ming Peng ${ }^{1}$,

6 Qin-Hui Cai ${ }^{1}$, Jian-Yi Yu ${ }^{5}$, Qun-Xiu Liu ${ }^{5}$, Zhao-Yang Liu ${ }^{8}$, Lin-Xiang Li ${ }^{9}$, Ming-Sheng Teng ${ }^{10}$,

7 Wei Huang ${ }^{11}$, Jun-Ying Zhou ${ }^{12}$, Chi Zhang ${ }^{13}$, Wu Chen ${ }^{1, *}$, Xiao-Long Tu ${ }^{2,3,4, *}$

8

30 \#Authors who made equal contribution to this article.

1. Guangzhou Zoo \& Guangzhou Wildlife Research Center, Guangzhou, 510070, China.

2. State Key Laboratory of Genetic Resources and Evolution, Kunming Institute of Zoology, Chinese Academy of Sciences, Kunming, 650201, China.

3. Kunming Natural History Museum of Zoology, Kunming Institute of Zoology, Chinese Academy of Sciences, Kunming, Yunnan, 650223, China.

4. Kunming College of Life Science, University of the Chinese Academy of Sciences, Kunming, 650204, China.

5. Shanghai Zoo, Shanghai, 200336, China.

6. CAAS-ILRI Joint Laboratory on Livestock and Forage Genetic Resources, Institute of Animal Science, Chinese Academy of Agricultural Sciences (CAAS), Beijing, 100193, China.

7. International Livestock Research Institute (ILRI), Nairobi, 00100, Kenya.

8. Wangcheng Park, Luoyang, 471000, China.

9. Suzhou Shangfangshan Forest Zoo, Suzhou, 215009, China.

10. Chongqing Zoo, Chongqing, 401326, China.

11. Nanchang Zoo, Nanchang, 330025, China.

12. Chinese Association of Zoological Gardens, Beijing, 100037, China.

13. Qinghai Province Key Laboratory of Crop Molecular Breeding, Key Laboratory of Adaptation and Evolution of Plateau Biota, Northwest Institute of Plateau Biology, Chinese Academy of Sciences, Xining, Qinghai, 810008, China. 
32 Abstract

33 The South China tigers (Panthera tigris amoyensis) are extinct in the wild, but viable

34 populations remain in breeding centers and zoos after 60 years of effective

35 conservation efforts. At present, however, the existing genetic variation of these tigers

36 remains unknown. In this study, we assembled a high-quality chromosome-level

37 genome using long-read sequences and re-sequenced 29 high-depth genomes of the

38 South China tigers. We identified two significantly differentiated genomic ancestries in

39 the extant populations, which also harbored some rare genetic variants introgressed

40 from other subspecies, suggesting limited but essential genetic diversity to sustain the

41 South China tigers. The unique pattern of dual ancestry and the genomic resources

42 generated in our study pay the way for a genomics-informed conservation, following

43 the real-time monitoring and controlled exchange of all reproductive South China

44 tigers. 


\section{Introduction}

5

The tiger (Panthera tigris) is one of the largest felids and a widely recognized flagship species of wildlife conservation in the world. There are six commonly accepted living tiger subspecies, including the Bengal tiger ( $P$. t. tigris), Amur tiger ( $P$. t. altaica), South China tiger (P. t. amoyensis), Sumatran tiger (P. t. sumatrae), Indochinese tiger (P. t. corbetti), and Malayan tiger (P. t. jacksoni $)^{1-3}$. Among them, the South China tiger is the rarest living subspecies and considered as critically endangered by the IUCN ${ }^{4}$. After their extinction in the wild, considerable efforts have been made to rescue them through captive breeding programs in China.

The Chinese Association of Zoological Gardens commenced a coordinated South China tiger captive breeding and management program in 1994, beginning with 47 tigers of 27 males and 20 females based on the studbook. Detailed pedigree records indicated that all South China tigers are descendants of six individuals, which were kept in the Shanghai Zoo (one male and two females) and the Guiyang Qianling Zoo (one male and two females) $^{5}$. In 2020, there were an estimated 205 South China tigers managed in captivity within China as well as 18 in the Laohu Valley Reserve in South Africa. Signatures of inbreeding such as reduced genetic diversity and low rate of successful breeding have been observed by analyzing the revised studbook of the captive populations of South China tigers ${ }^{6}$. In addition, the hybridization of South China tigers with other tiger subspecies was suggested based on a few mitochondrial and microsatellite markers ${ }^{7,8}$. Considering the positive correction between genetic 


\section{$84 \quad$ Results}

\section{tigers}

heterozygosity and fitness ${ }^{9,10}$ as well as the rapid fixation of one allele at individual genetic loci throughout the genomes following the increase in homozygosity and inbreeding load of the individuals in small, managed populations ${ }^{11,12}$, it is urgently needed to examine the genomic landscape of existing genetic variation that has driven the past successful rescue of the South China tigers, in reference to large-scale population genomic studies on other tiger subspecies ${ }^{1,13}$.

In this study, based on a de novo chromosome-level genome assembly and large-scale whole-genome re-sequencing data generated from extant South China tigers, we unexpectedly identified a relatively low genomic inbreeding buffered by two genetically differentiated ancestries in the major captive populations, which also retained some rare genetic variants following the past admixture from other subspecies. These findings not only explained the successful breeding history of the South China tigers in captivity, but also paved the way for the genomics-informed management by applying genome-wide markers to routinely monitor and sustain their critical genetic variation in the future.

\section{De novo genome assembly and whole-genome re-sequencing of the South China}

To investigate the genetic variation and evolution of South China tigers (Fig. 1a), we first constructed a high-quality de novo assembly of the South China tiger genome 
using a combination of high-fidelity short-read sequencing ${ }^{14}$, long-read

90 single-molecule real-time sequencing ${ }^{15}$, optical mapping ${ }^{16}$, and $\mathrm{Hi}^{-\mathrm{C}^{17}}$ technologies.

91 We generated a total of $122.31 \mathrm{~Gb}(50.96 \times)$ of PacBio long reads, $1,011.73 \mathrm{~Gb}$

$92(421.55 \times)$ of Illumina paired-end short reads, $440.32 \mathrm{~Gb}(183.47 \times)$ of BioNano optical

93 molecules, and $532.46 \mathrm{~Gb}(221.86 \times)$ of Hi-C data (Supplementary Tables S1 and S2).

$94 \mathrm{~K}-\mathrm{mer}^{18}$ analysis revealed its genome size to be $2.47 \mathrm{~Gb}$ (Supplementary Figure 1,

95 Supplementary Table S3). After being polished and finally corrected using the Illumina

96 short reads, the PacBio-based initial assembly resulted in a contig N50 at $6.20 \mathrm{Mb}$. We

97 scaffolded the PacBio contigs using the BioNano optical mapping data. The resulting

98 scaffolds were further clustered into chromosome-scale scaffolds using the Hi-C data

99 (Supplementary Figure 2). Finally, the de novo assembly generated $2.44 \mathrm{~Gb}$ of

100 genomic sequences with a contig N50 at $6.13 \mathrm{Mb}$ and a scaffold N50 at $150.19 \mathrm{Mb}$

101 (Fig. 1b, Supplementary Table S4). This novel assembly is the runner-up genome after

102 the one of African lion among all big cats and serves as the best reference genome of

103 tiger species up to today ${ }^{13,19-22}$. The de novo assembly contained 19

104 pseudochromosomes anchored with $2.40 \mathrm{~Gb}$ of contigs (99.35\%) and $2.42 \mathrm{~Gb}$ of

105 scaffolds (99.36\%), showing a high collinearity with the reference genome (FelCat9.0)

106 of the domestic cat (Felis catus) (Ensembl release 98, accessed in September 2019),

107 except for the E3 chromosome (Supplementary Figure 3, Supplementary Table S5).

$108 \mathrm{BUSCO}^{23}$ result demonstrated this novel genome to be $95.50 \%$ of its completeness

109 (Supplementary Table S6). By integrating the homology- and de novo-based

110 predictions, a total of $844.92 \mathrm{Mb}(34.98 \%)$ of repetitive elements and 20,910 
111 protein-coding genes were annotated (Supplementary Tables S7-S9). In addition, we

112 predicted non-coding RNA genes, including 568 microRNA (miRNA), 6,309 transfer

113 RNA (tRNA), 993 ribosomal RNA (rRNA), and 1,410 small nuclear RNA (snRNA)

114 genes (Supplementary Table S10). Altogether, we produced the first high-quality

115 chromosome-level assembly of the South China tiger genome (Supplementary Figures

1164 and 5).

117

118 To explore genetic variation lasted in the extant South China tigers, we re-sequenced

119 the whole genomes of 29 individuals collected from several major zoos in China. A

120 domestic cat was also re-sequenced and used as an outgroup (Fig. 1c). We generated

121 around 1,200 Gb of whole-genome sequencing data with an average coverage depth of

$12215.63 \times$. We combined our data with 40 previously published genomes of other five

123 tiger subspecies $(10.38-29.59 \times$ coverage depths) (Supplementary Figures 6 and 7,

124 Supplementary Tables S11-S13). We identified 10.21 million high-quality single

125 nucleotide polymorphisms (SNPs) in 69 tiger and one cat genomes after the stringent

126 quality control and the alignment of all re-sequencing data against our novel South

127 China tiger genome (Supplementary Figure 8, Supplementary Tables S14 and S15).

129 Two genomic ancestries sustained the extant South China tigers

130 To clarify the phylogenetic relationship and genetic background of different tiger

131 subspecies, we performed principal component analysis (PCA) (Supplementary

132 Figures 10 and 11) and a model-based ancestry estimation using ADMIXTURE 
133 software to infer their population genetic structure (Figure 2b, Supplementary Figure

134 9b). We found that all tiger subspecies were clearly diverged from each other, despite

135 potential gene flow among some of them, while the South China tigers were further

136 differentiated into two subgroups at the best $K=5$. We further reconstructed

137 neighbor-joining $(\mathrm{NJ})$ trees based on pairwise genetic distances with domestic cat as an

138 outgroup, which clearly supported the taxonomic status of six distinct living tiger

139 subspecies $^{1,3,24,25}$ (Fig. 2a, Supplementary Figure 9a). The maximum-likelihood tree

140 (Supplementary Figure 12) and identity-by-state analysis (Supplementary Figures

141 13-15) all verified this divergence pattern at subspecies level.

143 Based on the phylogenetic tree and the population genetic structure inferred by 144 ADMIXTURE, two clearly differentiated genomic ancestries (subgroups 1 and 2) were 145 identified among the South China tigers (Fig. 2a and 2b), a probable result of recent 146 but long assortative mating, as what was observed in the killer whale ecotypes ${ }^{26}$ and 147 highly inbred pigs $^{27}$, driving allelic segregation in the two tiger subgroups. The 148 inference by both MSMC2 (Fig. 2c) and $\delta a \delta i$ analyses (Supplementary Table S16) for 149 the potential scenario of these two ancestries suggested their initial segregation as early 150 as 3,000 years ago, while the divergence between the South China tiger and its closest 151 counterpart, the Amur tiger, was estimated around 20,000 years ago (Supplementary

152 Figure 16), to be highly similar to a previous study ${ }^{1}$. Thus, these findings confirmed 153 the presence of these two genomic ancestries among major South China tiger 154 populations. 
Limited gene flow from other tiger subspecies to extant South China tigers

157 To detect signal of potential genetic introgression from other tiger subspecies into the

158 South China tigers, we applied several population genetic analyses, including the 159 ABBA-BABA test ${ }^{28,29}, f 4-$ ratio $^{30}$, and TreeMix method ${ }^{31}$. The results clearly indicated 160 that the South China tiger sequenced by Liu et al. ${ }^{1}$ displayed the highest admixture 161 signal of hybrid origin from other subspecies (Fig. 2b and 2d). Although genetic 162 admixture occurred among tiger species (Supplementary Figures 17-19, 163 Supplementary Tables S17 and S18), we found a very low level of such introgression $164(0.13-1.50 \%)$ into the South China tiger genomes (Fig. 2d).

166 Pattern of genetic variation among six tiger subspecies

167 All tiger subspecies have been experiencing severe population bottlenecks due to 168 human hunting and habitat fragmentation. Here, we compared the levels of genetic 169 diversity and population differentiation among these subspecies. It was evident that the 170 Sumatran tigers showed the least genetic diversity in terms of both genome-wide 171 heterozygosity and nucleotide diversity $\left(\pi=0.553 \times 10^{-3}\right)($ Supplementary Figures 20

172 and 21, Supplementary Table S19) but the highest genetic differentiation from other 173 five tiger subspecies $\left(F_{\mathrm{ST}}=0.324-0.459\right)$ (Supplementary Table S20), confirming the 174 observations of previous studies ${ }^{1,13}$. Five Amur tigers had significantly higher 175 heterozygosity than the remaining six individuals (Fig. 3a), a clear genetic 176 sub-structuring within this subspecies due most likely to the recent admixture with 
177 other subspecies. However, the level of genetic diversity in all South China tiger

178 populations was not as low $\left(\pi=0.657 \times 10^{-3}\right)($ Supplementary Table S19) as what we

179 expected, following their pedigrees recorded in the studbook. Nonetheless, the South

180 China tigers also showed a significant genetic differentiation from other tiger

181 subspecies $\left(F_{\mathrm{ST}}=0.278-0.459\right)($ Supplementary Table S20).

183 We determined the relatedness among individuals within each tiger subspecies using

184 allelic identity-by-descent (IBD) ${ }^{26}$ and identified their different levels of inbreeding

185 (Fig. 3b, Supplementary Table S21). Further, we calculated individual inbreeding

186 based on whole genome SNPs using the inbreeding coefficients $F_{\mathrm{H}}$ (a measure of the

187 increase in individual SNP homozygosity compared with mean Hardy-Weinberg

188 expected homozygosity) and $F_{\mathrm{ROH}}{ }^{32}$ (based on runs of homozygosity (ROH) $>100 \mathrm{~kb}$ ).

189 Both analyses demonstrated the highest level of genome-wide inbreeding in the

190 Sumatran tigers, due probably to their founder event and strong recent bottleneck

191 (Supplementary Figure 22, Supplementary Table S22), while both Amur and South

192 China tigers had higher $F_{\mathrm{ROH}}$ values under longer $\mathrm{ROH}(1-2 \mathrm{Mb}$ and $>2 \mathrm{Mb})$, an

193 indication of their recent inbreeding/founder events and/or population

194 bottleneck/isolation (Fig. 3c). Although the pedigree-based inbreeding coefficients $\left(F_{\mathrm{p}}\right)$

195 of the South China tigers were calculated as high as $0.1796-0.5048^{5}$, the genomic IBD

196 was validated to be more strongly correlated with both $F_{\mathrm{H}}$ and $F_{\mathrm{ROH}}$ than $F_{\mathrm{p}}$ when

197 high-density SNPs are available ${ }^{33}$. The genome-wide SNP-based individual inbreeding

198 estimates from this study are thus recommended for assisting the decision-making of 
captive breeding of the South China tigers.

201 Because of the importance of managing deleterious mutations in conserved genomic

202 elements for species conservation ${ }^{34}$, we calculated the proportion of deleterious

203 mutations retained in potential coding regions of the six tiger subspecies and obtained

204 a total of 73,979 SNPs, $0.562 \%$ of which carried highly deleterious mutations, most of

205 them to be stop-gain, across all tiger subspecies (Supplementary Table S23). There was

206 no significant difference in high-, moderate-, and low-impact variants among the tiger

207 subspecies (Fig. 4a). The derived allele frequency distribution of high- and

208 moderate-impact mutations in the South China tigers did not display downward shifts

209 compared to low-impact mutations (nearly neutral) (Fig. 4b). Furthermore, the

210 numbers of homozygous sites with high-, moderate-, and low-impact mutations were

211 the lowest in the South China tigers compared with other subspecies (Fig. 4c,

212 Supplementary Table S24). Despite their inbreeding history, we did not observe the

213 accumulation of deleterious mutations or purging of highly deleterious mutations in the

214 South China tiger genomes, to be in a sharp contrast with many endangered mammals

215 experiencing severe bottlenecks and inbreeding ${ }^{35-37}$. This distinct distribution pattern of

216 deleterious mutations may have also facilitated the successful captive breeding and

217 rapid population recovery of the South China tigers.

\section{Discussion}

220 In the present study, we assembled a high-quality chromosome-level genome of the 
221 South China tiger, to provide a reference for further genetic and genomic studies of all 222 tigers. We found very limited genetic admixture from other tiger subspecies, 223 suggesting very little or even no genetic erosion in the South China tigers. This finding 224 contradicts previous opinions that "non-pure" tigers are caused by hybridization 7,8 . 225 However, we have only re-sequenced the whole genomes of 29 South China tigers 226 collected from four core breeding zoos in China, despite there are more than 200 South

227 China tigers today. As such, we shall continue and focus our efforts on collecting and 228 re-sequencing additional whole genomes of most, if not all, reproductive South China 229 tigers, to further fine-map and effectively manage their viable genomic landscapes 230 along with unique genetic variants present among their major populations.

232 Analysis of the different characteristics of genetic variation showed that, compared 233 with other tigers, the South China tigers harbored a moderate level of genetic diversity, 234 but escaped from the accumulation of harmful mutations in their conserved genomic 235 regions. We identified two genomic ancestries lasted in existing South China tiger 236 populations, as a probable outcome of recent segregation event and/or genetic drift 237 from limited founders being kept and reproduced in isolated zoos. However, we 238 inferred the differentiation time to be about 3,000 years ago. The Chinese Association 239 of Zoological Gardens has been coordinating the South China tiger captive breeding 240 and management program since 1994. All South China tigers are considered as the 241 descendants of one wild-caught tigress from Fujian and five wild-caught tigers from 242 Guizhou between 1958 and $1970^{5}$. Our results clearly corroborated the existence of 
243 these two lineages. We therefore recommend a genomics-informed management of 244 these two genetic ancestries, to minimize a further loss of the unique and critical 245 genetic variants but a potential increase in the inbreeding load across all South China 246 tiger populations. All the new findings from our present study demonstrate the power 247 and effectiveness of concerted efforts to conserve the South China tigers in the past 248 and shed light into a potentially bright future of these critically endangered cats, to be 249 another case as what is achieved in the conservation of Giant Panda to be off the 250 endangered list of $\mathrm{IUCN}^{4}$.

\section{$252 \quad$ Materials and methods}

\section{De novo genome sequencing}

254 Blood samples of one male (Tuantuan) and another female (Huanhuan) South China 255 tiger were acquired from Guangzhou Zoo for genome sequencing. All libraries were 256 constructed at BGI (Shenzhen, China). Three short insert libraries (270, 500, and 800 $257 \mathrm{bp})$ were constructed for Tuantuan and four long mate-paired insert libraries $(2,5,10$, 258 and $20 \mathrm{~kb}$ ) were constructed for Huanhuan. All libraries were sequenced on the 259 Illumina HiSeq $X$ Ten Platform using 150-bp paired-end reads according to the 260 Illumina protocols, except for the 800-bp library, which was sequenced on the 261 HiSeq2500 System using 125-bp paired-end reads. Genomic DNA from the Tuantuan 262 blood sample was also used to prepare libraries for sequencing on a PacBio RS II 263 sequencer. 
266 We applied 17-mer distribution analysis to estimate the tiger genome size using clean

267 reads from the short-insert libraries. Genome size was calculated using the formula:

268 Genome size = K-mer_num / Peak_depth.

270 Genome assembly

271 The PacBio data were de novo assembled using WTDBG-1.2.8

272 (https://github.com/ruanjue/wtdbg-1.2.8) and genomic contigs were polished with the

273 Arrow program (https://www.pacb.com/support/software-downloads/) by aligning

274 SMRT reads, which yielded an assembly (2.43 Gb in length) with N50 size at $6.24 \mathrm{Mb}$.

275 We also aligned the Illumina X Ten data to the assembly using BWA (v0.7.15) ${ }^{38}$ for

276 error correction by Pilon $(\mathrm{v} 1.22)^{39}$ with the parameter '--mindepth 6', which is an

277 integrated tool for comprehensive variant detection and genome assembly

278 improvement. The final assembly generated a total length of $2.42 \mathrm{~Gb}$ and N50 length at $279 \quad 6.20 \mathrm{Mb}$.

\section{Construction of optical genome maps}

282 DNA of sufficient quality was extracted and labelled from the Tuantuan blood cells 283 according to standard BioNano protocols (BioNano Genomics), after which nicking, 284 labeling, repairing, and staining processes were implemented. We digested DNA using 285 a specific single-stranded nicking endonuclease (Nt.BspQI). BioNano Solve (v3.0.1) ${ }^{40}$ 286 was used to produce optical maps with single molecules above $100 \mathrm{~kb}$ in size and six 


\section{Hi-C analysis}

290 The Hi-C library was constructed from the blood cells of a male South China tiger 291 (Kangkang) according to the standard procedures of BGI and sequenced using the 292 MGISEQ-2000 Platform. The BioNano-based scaffolds were anchored into a 293 chromosome-scale assembly using a Hi-C proximity-based assembly approach. We 294 aligned the Hi-C reads to scaffolds using bowtie2 (v2.2.5) ${ }^{41}$ and interaction maps were 295 generated following HiC-Pro $(\mathrm{v} 2.5 .0)^{42}$ pipelines. The uniquely mapped read pairs 296 were used as input for Juicer ${ }^{43}$ and $3 \mathrm{~d}_{-}-\mathrm{DNA}^{44} \mathrm{Hi}-\mathrm{C}$ analysis and scaffolding pipelines.

297 The resulting Hi-C contact maps were visualized using Juicebox ${ }^{45}$, and mis-assemblies 298 and mis-joins were manually corrected based on neighboring interactions. The final 299 chromosome assembly was then generated.

301 Genome annotation

302 We identified repetitive sequences in the genomes using a combination of homology303 and de novo-based methods. First, RepeatMasker ${ }^{46}$ and RepeatProteinMask ${ }^{46}$ were 304 used to search repeat sequences against with Repbase database ${ }^{47,48}$. Second, 305 LTRharvest (GenomeTools v1.5.9) $)^{49} \quad$ and $\quad$ RepeatModeler 306 (http://www.repeatmasker.org/RepeatModeler/) were used to build a de novo repeat 307 library, and then repeats were annotated using RepeatMasker ${ }^{46}$ with default parameters. 308 Last, tandem repeats were detected using Tandem Repeats Finder (TRF) ${ }^{50}$. 
310 For gene structure prediction, we used homology-based prediction based on the protein

311 sequences from five species (Felis catus, Homo sapiens, Mus musculus, Panthera

312 pardus, and Panthera tigris altaica) downloaded from the Ensembl database (release

313 93). These protein sequences were mapped to the South China tiger genome using

314 TBLASTN (E-value cutoff: $\left.1 \mathrm{e}^{-5}\right)^{51}$. High-scoring segment pairs (HSPs) were

315 concatenated using Solar $(\mathrm{v} 0.9 .6)^{18}$. GeneWise $(\mathrm{v} 2.4 .1)^{52}$ was used to define accurate

316 gene models. We then merged and filtered redundancy from different homology results

317 based on the GeneWise score $(\geq 0.4)$. To obtain the final gene set, transposons and

318 single-exon genes without functional annotations were filtered out.

320 Gene functional annotations were assigned using BLASTP (BLAST+ v2.2.26) ${ }^{51}$

321 against public databases, including the Swiss-Prot (release-2017_09) ${ }^{53}$, TrEMBL

322 (release-2017_09) $)^{53}$, KEGG (v84.0) $)^{54}, \mathrm{COG}^{55}$, and NCBI nucleotide collection $\mathrm{nr} / \mathrm{nt}$

323 (https://www.ncbi.nlm.nih.gov/nucleotide/, v20170924). The motifs and domains in the

324 protein sequences were annotated using InterProScan (v5.16-55.0) $)^{56}$.

326 The tRNA genes were predicted using tRNAscan-SE (v1.3.1) ${ }^{57}$ with eukaryote

327 parameters. The rRNA fragments were identified by aligning the rRNA template 328 sequences from the Human Rfam database ${ }^{58}$ using BLASTN (BLAST+ v2.2.26) ${ }^{51}$ 329 (E-value 1e-5). The snRNAs and miRNAs were searched via a two-step method: i.e., 330 aligned with BLAST and then searched with INFERNAL (infernal-1.1.1) ${ }^{59}$ against the 
Rfam database.

333 Sampling information and genome re-sequencing. We collected a total of 30 334 specimens, including 29 South China tigers (P. t. amoyensis) from several major zoos 335 in China and a domestic cat (Felis catus) from Guangzhou Zoo, China (Supplementary 336 Table S11). Genomic DNA was extracted from whole blood using the DNeasy Blood

$337 \&$ Tissue Kit (QIAGEN, Valencia, California, USA) following the manufacturer's 338 protocols. Sequencing libraries were prepared according to the Illumina library 339 preparation pipeline and sequenced using the Illumina Hiseq $\mathrm{X}$ Ten platform to 340 generate $150-$ bp paired-end reads.

342 Quality control. To ensure reads were reliable and without artificial bias (e.g., 343 low-quality paired reads that result from base-calling duplicates and adapter 344 contamination), we conducted a series of quality control (QC) procedures, as follows:

345 (1) Removed reads with $\geq 1 \%$ unidentified nucleotides $(\mathrm{N})$.

346 (2) Removed reads with $>40 \%$ bases having phred quality $<20$.

347 (3) Removed reads with $>10$ nucleotides overlapping the adapter (allowing $\leq 10 \%$ 348 mismatches).

350 Read alignment and variant calling. We used $\mathrm{BWA}^{60}$ to align the clean reads of each 351 sample against our newly assembled South China tiger genome (settings: mem -t 4 -k $35232-\mathrm{M}-\mathrm{R}$ ). Alignment files were converted to BAM files using SAMtools (settings: 
$353-\mathrm{bS}-\mathrm{t})(\mathrm{v}-0.1 .19)^{61}$. In addition, potential PCR duplications were removed using 354 Picard (http://broadinstitute.github.io/picard/). We called SNPs using the 355 HaplotypeCaller approach implemented in the Genome Analysis Toolkit (GATK) 356 package ${ }^{62}$. Filtering criteria were as follows:

357 (1) SNPs with (QD < 2.0; FS > 60.0; MQ < 40.0; QUAL < 30; DP < 4.0; MQRankSum $358<-12.5 ;$ ReadPosRankSum $<-8.0)$ were filtered.

359 (2) Multi-nucleotide polymorphisms were ignored.

360 (3) SNPs within 5 bp of a gap were filtered.

361 (4) Overall depth (for all individuals) was $>1 / 3 \times$ and $<3 \times$.

362 (5) Unobserved variant allele constituted $<10 \%$.

363 A total of 54,067,600 high-quality SNPs were retained for subsequent analyses after 364 filtering. Gene-based SNP annotation was performed using ANNOVAR ${ }^{63}$.

Phylogenetic and population genetic analysis. We selected whole-genome SNPs and

367 four-fold-degenerate sites (4D-sites) that represent neutral or near-neutral variants for 368 phylogenetic and population structure analyses. PCA was performed using PLINK $369(\mathrm{v} 1.9)^{64}$. Genetic structure was inferred using ADMIXTURE (v1.3) $)^{65}$, with 370 implementation of a block-relaxation algorithm. To explore convergence of individuals, 371 we predefined the number of genetic clusters $K$ from 1 to 9 . We calculated the $372 p$-distance matrix using VCF2Dis (https://github.com/BGI-shenzhen/VCF2Dis) and a 373 neighbor-joining tree was generated using the R package $\mathrm{APE}^{66}$. 
375 Calculation of genetic diversity. Pairwise nucleotide diversity $\theta_{\pi}$ and Watterson's 376 estimator $\theta_{\mathrm{w}}{ }^{67}$ within a tiger subspecies were calculated using a sliding-window approach (20-kb windows sliding in 10-kb steps). Genetic differentiation between tiger

378 subspecies was calculated using the pairwise fixation index $F_{\mathrm{ST}^{68}}$.

380 Identity-by-state (IBS) and identity-by-descent (IBD) analyses. To evaluate the

381 similarity between two tigers within a subspecies, genome-wide IBS pairwise identities

382 were calculated using the toolset SNPRelate ${ }^{69}$ in the R package, based on the matrix of 383 genome-wide IBS pairwise distances, we performed multidimensional scaling analysis 384 and cluster analysis, and determined the groups using a permutation score. We also 385 calculated the pairwise IBD using PLINK (v1.9) ${ }^{64}$.

387 Introgression and demographic history analyses. We separated the South China 388 tigers into three classes (i.e., subgroup 1, subgroup 2, and ptam1) based on phylogeny. 389 TreeMix (v1.13) ${ }^{70}$ was used to detect gene flow between the tiger subspecies based on 390 genome-wide allele frequency data at individual SNPs. We first constructed a 391 maximum-likelihood tree for the subspecies using blocks of 10,000 SNPs. The number 392 of migration events was set from 1 to 6 . We calculated introgression among the tiger 393 subspecies using Patterson's D-statistic (ABBA-BABA test) ${ }^{71}$, with the cat as the 394 outgroup, and tested the proportions of admixture events (f4-ratio) within each South 395 China tiger using Dsuite $(\mathrm{v} 0.4)^{30}$. 
397 We used the PSMC ${ }^{72}$ model to reconstruct demographic history. To estimate effective 398 ancestral population size changes for individual tiger subspecies, we selected 399 individuals with high sequencing depth to ensure the quality of the consensus sequence. 400 SNPs were detected using SAMtools ${ }^{61}$, sites were filtered based on a minimum depth $401(\mathrm{DP}=4)$ or high depth $(\mathrm{DP}=50)$ and mapping quality $(\mathrm{Q}=20)$. We only retained 402 autosomal SNPs. Parameters were set to: $-\mathrm{N} 30$, -t15, -r5, $-\mathrm{p}^{`} 4+25^{*} 2+4+6$ ’.

$404 \mathrm{MSMC2}^{73}$ was used to infer population separation history. To obtain a greater 405 resolution, we estimated divergence times between the two South China tiger 406 subgroups using eight haplotypes per group. We also applied this method to determine 407 divergence between the South China tigers and each of other tiger subspecies using 408 two haplotypes per group.

$410 \delta a \delta \mathrm{i}^{74}$ analysis was further employed to detect the differentiation time of the two South

411 China tiger subgroups. Only SNPs in intergenic regions were remained to ensure their 412 neutrality. We imputed ungenotyped markers and phased genotypes using BEAGLE ${ }^{75}$. 413 Besides, SNPs with MAF $<0.01$ were filtered. The model with the maximum 414 log-likelihood value was considered as the optimal one. A mutation rate of $3.5 \times 10^{-9}$ 415 per base per generation and generation time of 5 years was used.

417 Analysis of runs of homozygosity (ROH). Regions of homozygosity were extracted 418 for all chromosomes of all individuals based on SNP information. PLINK (v1.9) ${ }^{64}$ was 
419 used to detect $\mathrm{ROH}$ via a sliding window approach, with the following parameters:

420 '--homozyg-window-snp 100 --homozyg-window-het 2 --homozyg-window-missing 5

421 --homozyg-snp 100 --homozyg-kb 100 --homozyg-density 10 --homozyg-gap 100’.

422

423 Inbreeding coefficient. We measured individual inbreeding using the genomic 424 inbreeding coefficients $F_{\mathrm{H}}^{33}$, which is the fraction of IBD of the two alleles in a diploid 425 individual from a common ancestor. $F_{\mathrm{H}}$ was calculated using PLINK (v1.9) ${ }^{64}$. 426 Alternatively, individual genomic inbreeding coefficients was also measured using $427 \quad F_{\mathrm{ROH}}{ }^{76}$, which is an estimate of $\mathrm{ROH}$ proportion in an individual genome.

429 Identification of deleterious mutations. The variants leading to functional changes 430 were regarded as candidates of deleterious mutations. Thus, we only analyzed all 431 potential coding region SNPs. SnpEff (v4.3t) ${ }^{77}$ was used for genetic variant annotation 432 and functional effect prediction.

\section{Ethics declarations}

435 All necessary research permits and ethics approvals for this study were granted by 436 Guangzhou Zoo (Project number GZ_20180216).

Data and material availability: Genome assemblies and DNA sequencing data were

439 deposited in the GSA database under Project no. PRJCA006384. The de novo genome 440 of the South China tiger was deposited under Accession ID GWHBEIN00000000. The 
441 re-sequencing genomic data of 29 South China tigers and a domestic cat were 442 deposited under Accession ID CRA004909.

\section{References}

4451 Liu, Y. C. et al. Genome-wide evolutionary analysis of natural history and 446 adaptation in the world's tigers. Curr Biol 28, 3840-3849 e3846, 447 doi:10.1016/j.cub.2018.09.019(2018).

4482 Luo, S. J. et al. Phylogeography and genetic ancestry of tigers (Panthera tigris). $449 \quad$ PLoS Biol 2, e442, doi:10.1371/journal.pbio.0020442 (2004).

4503 Luo, S. J., Liu, Y. C. \& Xu, X. Tigers of the world: genomics and conservation. 451 Annu Rev Anim Biosci 7, 521-548, doi:10.1146/annurev-animal-020518-115106 $452 \quad(2019)$.

4534 IUCN. The IUCN red List of threatened species. Version 2021-2. 454 https://www.iucnredlist.org (2021).

4555 Yin, Y. Z. Studbook of the South China tiger. (Chongqing Zoological Garden, 456 China, 2016).

4576 Tilson, R., Traylor-Holzer, K. \& Jiang, Q. M. The decline and impending extinction 458 of the South China tiger. Oryx 31, 243-252, 459 doi:10.1046/j.1365-3008.1997.d01-123.x (2009).

4607 Guo, J. Year of the tiger. Nature 449, 16-18, doi:10.1038/449016a (2007).

4618 Zhang, W. et al. Sorting out the genetic background of the last surviving South 462 China Tigers. Journal of Heredity 110, 641-650, doi:10.1093/jhered/esz034 (2019). 
9 Reed, D. H. \& Frankham, R. Correlation between fitness and genetic diversity. Conserv Biol 17, 230-237, doi:10.1046/j.1523-1739.2003.01236.x (2003).

10 Vandewoestijne, S., Schtickzelle, N. \& Baguette, M. Positive correlation between genetic diversity and fitness in a large, well-connected metapopulation. BMC Biol 6, 46, doi:10.1186/1741-7007-6-46 (2008).

11 Keller, L. F. \& Waller, D. M. Inbreeding effects in wild populations. Trends in Ecology \& Evolution 17, 230-241, doi:10.1016/S0169-5347(02)02489-8 (2002).

12 Hedrick, P. W. \& Garcia-Dorado, A. Understanding inbreeding depression, purging, and genetic rescue. Trends Ecol Evol 31, 940-952, doi:10.1016/j.tree.2016.09.005 (2016).

13 Armstrong, E. E. et al. Recent evolutionary history of tigers highlights contrasting roles of genetic drift and selection. Mol Biol Evol 38, 2366-2379, doi:10.1093/molbev/msab032 (2021).

14 Paszkiewicz, K. \& Studholme, D. J. De novo assembly of short sequence reads. Brief Bioinform 11, 457-472, doi:10.1093/bib/bbq020 (2010).

15 Eid, J. et al. Real-time DNA sequencing from single polymerase molecules. Science 323, 133-138, doi:10.1126/science.1162986 (2009).

16 Schwartz, D. C. et al. Ordered restriction maps of Saccharomyces cerevisiae chromosomes constructed by optical mapping. Science 262, 110-114, doi:10.1126/science.8211116 (1993).

17 Lieberman-Aiden, E. et al. Comprehensive mapping of long-range interactions reveals folding principles of the human genome. Science 326, 289-293, 
$18 \mathrm{Li}$, R. et al. The sequence and de novo assembly of the giant panda genome. Nature 463, 311-317, doi:10.1038/nature08696 (2010).

19 Armstrong, E.E., Taylor, R.W., Miller, D.E. et al. Long live the king: chromosome-level assembly of the lion (Panthera leo) using linked-read, Hi-C, and long-read data. BMC Biol 18, doi:10.1186/s12915-019-0734-5 (2020).

20 Cho, Y. S. et al. The tiger genome and comparative analysis with lion and snow leopard genomes. Nat Commun 4, 2433, doi:10.1038/ncomms3433 (2013).

$21 \mathrm{Kim}, \mathrm{S}$. et al. Comparison of carnivore, omnivore, and herbivore mammalian 
doi:10.1371/journal.pone.0004125 (2009).

26 Foote, A. D. et al. Genome-culture coevolution promotes rapid divergence of killer whale ecotypes. Nat Commun 7, 11693, doi:10.1038/ncomms11693 (2016).

27 Wang, L. G. et al. Genomic analysis reveals specific patterns of homozygosity and heterozygosity in inbred pigs. Animals 9, 314, doi:10.3390/ani9060314 (2019).

28 Zeng, L. et al. Genomes reveal selective sweeps in kiang and donkey for high-altitude $\quad$ adaptation. $\quad$ Rool 42, 450-460, doi:10.24272/j.issn.2095-8137.2021.095 (2021).

29 Green, R. E. et al. A draft sequence of the Neandertal genome. Science 328, 710-722, doi:10.1126/science.1188021 (2010).

30 Malinsky, M., Matschiner, M. \& Svardal, H. Dsuite - Fast D-statistics and related admixture evidence from VCF files. Mol Ecol Resour 21, 584-595, doi:10.1111/1755-0998.13265 (2021).

31 Zeng, L. et al. Whole genomes and transcriptomes reveal adaptation and domestication of pistachio. Genome Biol 20, 79, doi:10.1186/s13059-019-1686-3 (2019).

32 Kardos, M. et al. Genomic consequences of intensive inbreeding in an isolated wolf population. Nat Ecol Evol 2, 124-131, doi:10.1038/s41559-017-0375-4 (2018).

33 Kardos, M., Luikart, G. \& Allendorf, F. W. Measuring individual inbreeding in the age of genomics: marker-based measures are better than pedigrees. Heredity 115, 63-72, doi:10.1038/hdy.2015.17 (2015). 
34 van Oosterhout, C. Mutation load is the spectre of species conservation. Nat Ecol Evol 4, 1004-1006, doi:10.1038/s41559-020-1204-8 (2020).

35 Grossen, C., Guillaume, F., Keller, L. F. \& Croll, D. Purging of highly deleterious mutations through severe bottlenecks in Alpine ibex. Nature Commun 11, doi:100110.1038/s41467-020-14803-1 (2020).

36 Feng, S. et al. The genomic footprints of the fall and recovery of the Crested Ibis. Curr Biol 29, 340-349 e347, doi:10.1016/j.cub.2018.12.008 (2019).

37 Xue, Y. et al. Mountain gorilla genomes reveal the impact of long-term population decline and inbreeding. Science 348, 242-245, doi:10.1126/science.aaa3952 (2015).

38 Li, H. \& Durbin, R. Fast and accurate short read alignment with Burrows-Wheeler transform. Bioinformatics 25, 1754-1760, doi:10.1093/bioinformatics/btp324 (2009).

39 Walker, B. J. et al. Pilon: an integrated tool for comprehensive microbial variant detection and genome assembly improvement. PLoS One 9, e112963, doi:10.1371/journal.pone.0112963 (2014).

40 Lam, E. T. et al. Genome mapping on nanochannel arrays for structural variation analysis and sequence assembly. Nat Biotechnol 30, 771-776, doi:10.1038/nbt.2303 (2012).

41 Langmead, B. \& Salzberg, S. L. Fast gapped-read alignment with Bowtie 2. Nat Methods 9, 357-359, doi:10.1038/nmeth.1923 (2012).

42 Servant, N. et al. HiC-Pro: an optimized and flexible pipeline for $\mathrm{Hi}-\mathrm{C}$ data processing. Genome Biol 16, 259, doi:10.1186/s13059-015-0831-x (2015). 
43 Durand, N. C. et al. Juicer provides a one-click system for analyzing loop-resolution Hi-C experiments. Cell Syst 3, 95-98, doi:10.1016/j.cels.2016.07.002 (2016).

44 Dudchenko, O. et al. De novo assembly of the Aedes aegypti genome using Hi-C Acids Res 27, 573-580, doi:10.1093/nar/27.2.573 (1999).

57251 Altschul, S. F., Gish, W., Miller, W., Myers, E. W. \& Lipman, D. J. Basic local 
alignment search tool. $J$ Mol Biol 215, 403-410, doi:10.1016/S0022-2836(05)80360-2 (1990).

52 Birney, E., Clamp, M. \& Durbin, R. GeneWise and Genomewise. Genome Res 14, 988-995, doi:10.1101/gr.1865504 (2004).

53 Boeckmann, B. et al. The SWISS-PROT protein knowledgebase and its supplement TrEMBL in 2003. Nucleic Acids Res 31, 365-370, doi:10.1093/nar/gkg095 (2003).

54 Kanehisa, M. \& Goto, S. KEGG: kyoto encyclopedia of genes and genomes. Nucleic Acids Res 28, 27-30, doi:10.1093/nar/28.1.27 (2000).

55 Tatusov, R. L. et al. The COG database: an updated version includes eukaryotes. BMC Bioinformatics 4, 41, doi:10.1186/1471-2105-4-41 (2003).

56 Mulder, N. \& Apweiler, R. InterPro and InterProScan: tools for protein sequence classification and comparison. Methods Mol Biol 396, 59-70, doi:10.1007/978-1-59745-515-2_5(2007).

57 Lowe, T. M. \& Eddy, S. R. tRNAscan-SE: a program for improved detection of transfer RNA genes in genomic sequence. Nucleic Acids Res 25, 955-964, doi:10.1093/nar/25.5.955 (1997).

58 Griffiths-Jones, S. et al. Rfam: annotating non-coding RNAs in complete genomes. Nucleic Acids Res 33, D121-124, doi:10.1093/nar/gki081 (2005).

59 Nawrocki, E. P., Kolbe, D. L. \& Eddy, S. R. Infernal 1.0: inference of RNA alignments. Bioinformatics 25, 1335-1337, doi:10.1093/bioinformatics/btp157 (2009). 
$60 \mathrm{Li}, \mathrm{H}$. Aligning sequence reads, clone sequences and assembly contigs with BWA-MEM. arXiv, arXiv:1303.3997 (2013).

$61 \mathrm{Li}, \mathrm{H}$. et al. The Sequence Alignment/Map format and SAMtools. Bioinformatics 25, 2078-2079, doi:10.1093/bioinformatics/btp352 (2009).

62 McKenna, A. et al. The Genome Analysis Toolkit: a MapReduce framework for analyzing next-generation DNA sequencing data. Genome Res 20, 1297-1303, doi:10.1101/gr.107524.110 (2010).

63 Wang, K., Li, M. \& Hakonarson, H. ANNOVAR: functional annotation of genetic variants from high-throughput sequencing data. Nucleic Acids Res 38, e164, doi:10.1093/nar/gkq603 (2010).

64 Purcell, S. et al. PLINK: a tool set for whole-genome association and population-based linkage analyses. Am $J$ Hum Genet 81, 559-575, doi:10.1086/519795 (2007).

65 Alexander, D. H., Novembre, J. \& Lange, K. Fast model-based estimation of ancestry in unrelated individuals. Genome Res 19, 1655-1664, doi:10.1101/gr.094052.109 (2009).

66 Paradis, E., Claude, J. \& Strimmer, K. APE: Analyses of phylogenetics and evolution in $\mathrm{R}$ language. Bioinformatics 20, 289-290, doi:10.1093/bioinformatics/btg412 (2004).

67 Nei, M. \& Li, W. H. Mathematical model for studying genetic variation in terms of restriction endonucleases. Proc Natl Acad Sci $U$ S A 76, 5269-5273, doi:10.1073/pnas.76.10.5269 (1979). 
61768 Weir, B. S. \& Cockerham, C. C. Estimating F-statistics for the analysis of 618 population $\quad$ structure. $\quad$ Evolution $\quad 38, \quad$ 1358-1370, 619 doi:10.1111/j.1558-5646.1984.tb05657.x (1984).

62069 Zheng, X. et al. A high-performance computing toolset for relatedness and 621 principal component analysis of SNP data. Bioinformatics 28, 3326-3328, 622 doi:10.1093/bioinformatics/bts606 (2012).

62370 Pickrell, J. K. \& Pritchard, J. K. Inference of population splits and mixtures from 624 genome-wide allele frequency data. PLoS Genet 8, e1002967, 625 doi:10.1371/journal.pgen.1002967 (2012).

62671 Patterson, N. et al. Ancient admixture in human history. Genetics 192, 1065-1093, 627 doi:10.1534/genetics.112.145037 (2012).

$62872 \mathrm{Li}, \mathrm{H}$. \& Durbin, R. Inference of human population history from individual 629 whole-genome sequences. Nature 475, 493-496, doi:10.1038/nature10231 (2011). 63073 Schiffels, S. \& Durbin, R. Inferring human population size and separation history 631 from multiple genome sequences. Nat Genet 46, 919-925, doi:10.1038/ng.3015 $632 \quad$ (2014)

63374 Gutenkunst, R. N., Hernandez, R. D., Williamson, S. H. \& Bustamante, C. D. 634 Inferring the joint demographic history of multiple populations from 635 multidimensional SNP frequency data. PLoS Genet 5, e1000695, 636 doi:10.1371/journal.pgen.1000695 (2009).

63775 Browning, S. R. \& Browning, B. L. Rapid and accurate haplotype phasing and 638 missing-data inference for whole-genome association studies by use of localized 

doi:10.1086/521987 (2007).

76 Kardos, M., Qvarnstrom, A. \& Ellegren, H. Inferring individual inbreeding and demographic history from segments of identity by descent in Ficedula flycatcher genome sequences. Genetics 205, 1319-1334, doi:10.1534/genetics.116.198861 (2017).

77 Cingolani, P. et al. A program for annotating and predicting the effects of single nucleotide polymorphisms, SnpEff: SNPs in the genome of Drosophila melanogaster strain w1118; iso-2; iso-3. Fly (Austin) 6, 80-92, doi:10.4161/fly.19695 (2012).

\section{Acknowledgments}

We thank Z.J.C., A.P.J., Q.R.W., and X.J.C. from Guangzhou Zoo for providing support during this study. This work was supported by the Science and Technology Program of Guangzhou, China (202102020815).

\section{Author contributions}

W.C., X.L.T., C.W., and D.D.W. led the project. C.W., X.L.T., and D.D.W. drafted the

657 manuscript. X.L.T, D.D.W., and M.C.Y. performed data analysis. W.C., Y.H.Y., 658 Y.J.W., M.H., S.M.P., Q.H.C., F.S., J.Y.Y., Q.X.L., Z.Y.L., L.X.L., M.S.T., W.H., version of the manuscript. 
662 Additional information

663 Competing interests: The authors declare no competing financial interests. 

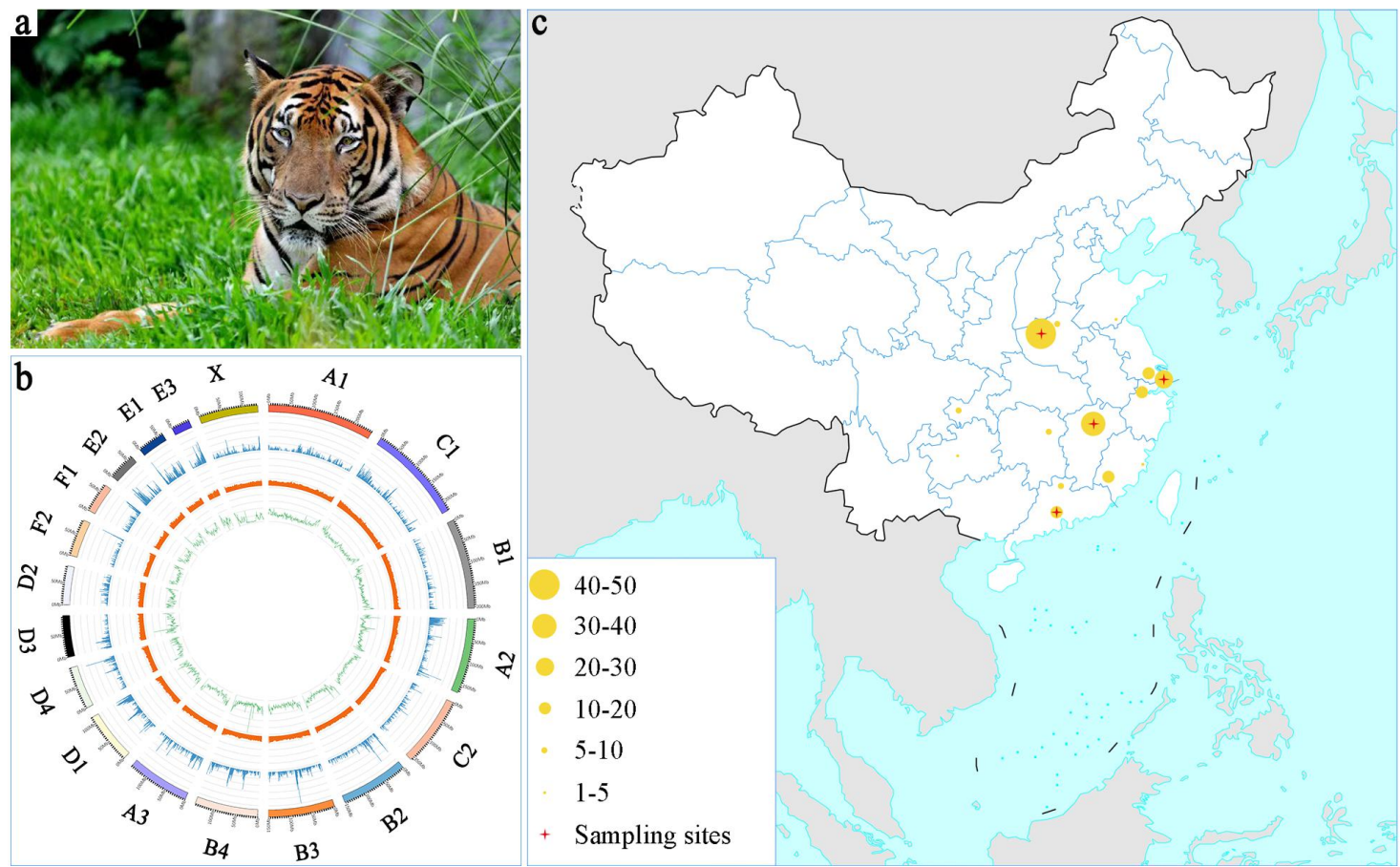

Sampling sites

Fig. 1 | Genome of South China tiger. (a) A male South China tiger. (b) Circos plot of South

667 China tiger genomic features. Outer to inner: pseudochromosome, gene density $(500-\mathrm{kb}$

668 window) (with higher gene density indicated by higher blue column), GC content (500-kb

669 window), and SNP density (500-kb window). (c) Distribution of captive South China tigers in

670 China. Yellow circles show 15 city locations of captive South China tigers, including Luoyang,

671 Zhengzhou, Linyi, Suzhou, Shanghai, Hangzhou, Nanchang, Chongqing, Chengdu, Guiyang,

672 Changsha, Fuzhou, Longyan, Shaoguan, and Guangzhou. Circle size is proportional the

673 number of South China tigers in each city. All data are from South China tiger studbook (2020).

674 Red cross represents sampling site of the South China tigers in our study. 

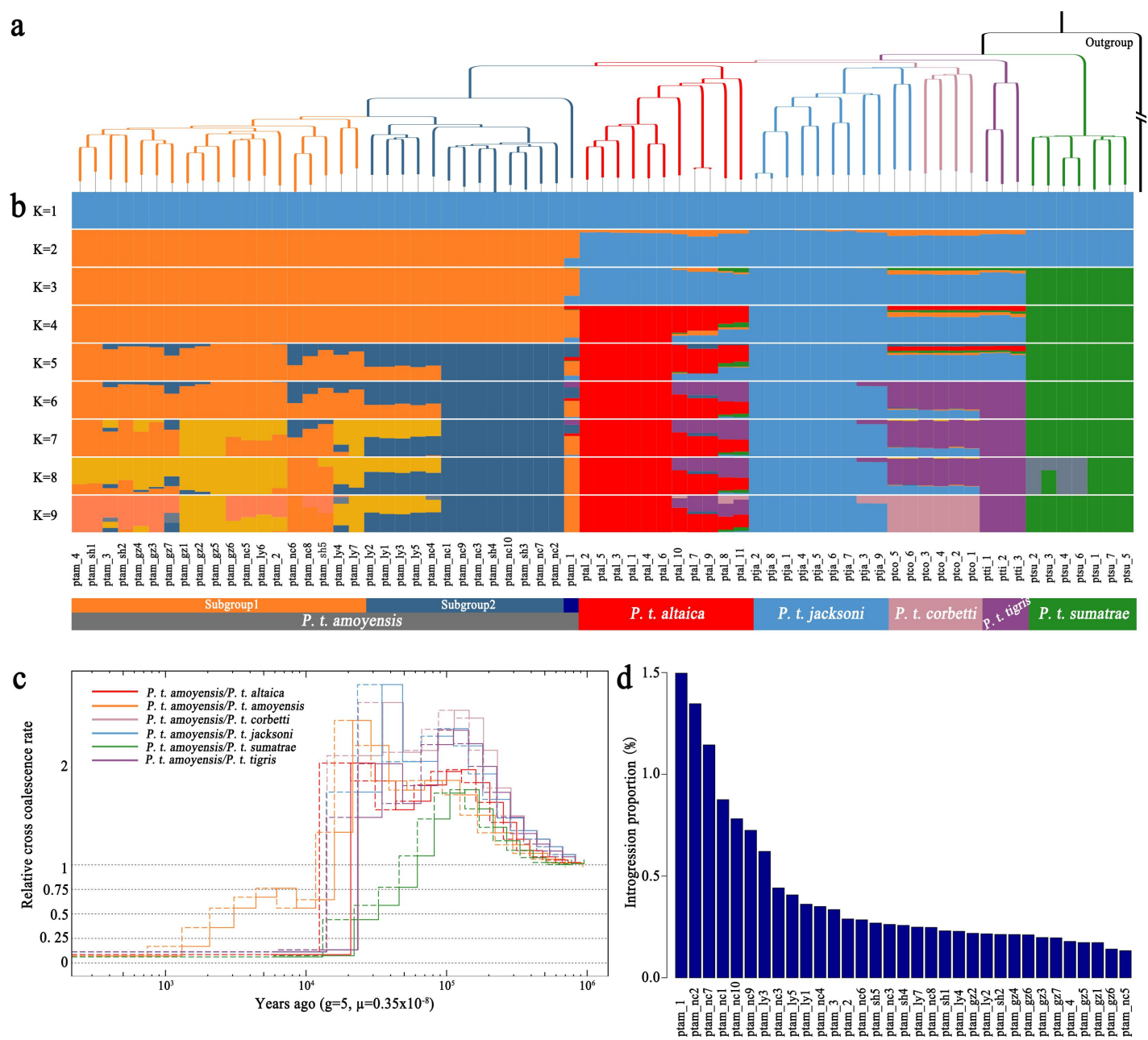

Fig. 2 | Genetic components of extant South China tigers. (a) Phylogenetic relationship of

677 the South China tigers with other tiger subspecies, with a domestic cat as an outgroup. (b)

678 Population genetic structuring of different tiger subspecies. (c) Differentiation time between

679 two sub-populations among the South China tigers (subgroups 1 and 2) and divergence time

680 between the South China tigers and other tiger subspecies inferred by MSMC2. (d) Average

681 ratio of genetic components between the South China tiger and other subspecies. 

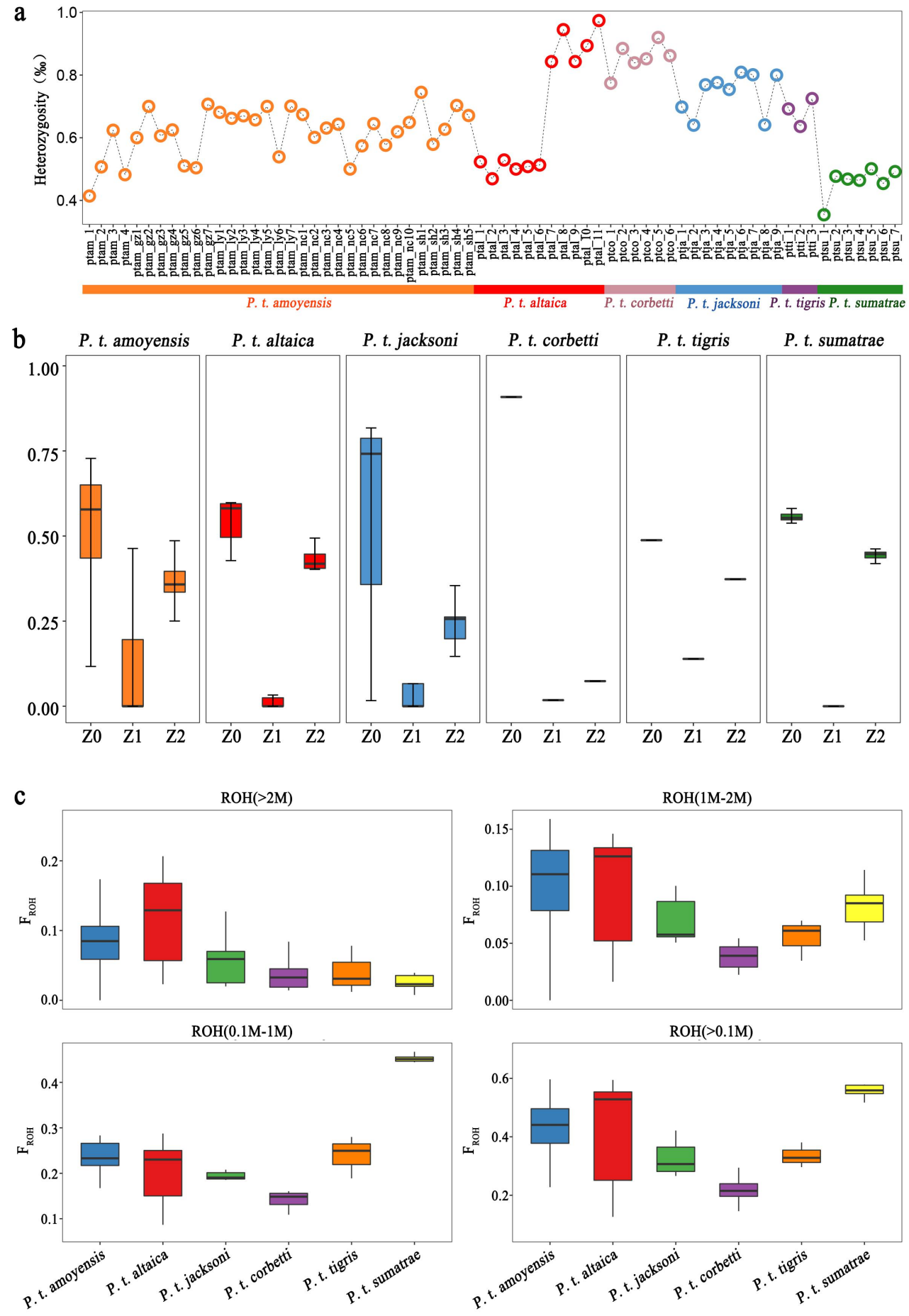

683 Fig. 3 | Comparison of genetic variation among different tiger subspecies. (a)

684 Genome-wide heterozygosity per individual. (b) Pairwise relatedness based on allelic 685 identity-by-descent (IBD). (c) Genomic inbreeding coefficients $\left(F_{\mathrm{ROH}}\right)$ based on different 686 lengths of runs of homozygosity $(\mathrm{ROH})$, with a minimum length of $100 \mathrm{~kb}$. 
a


c

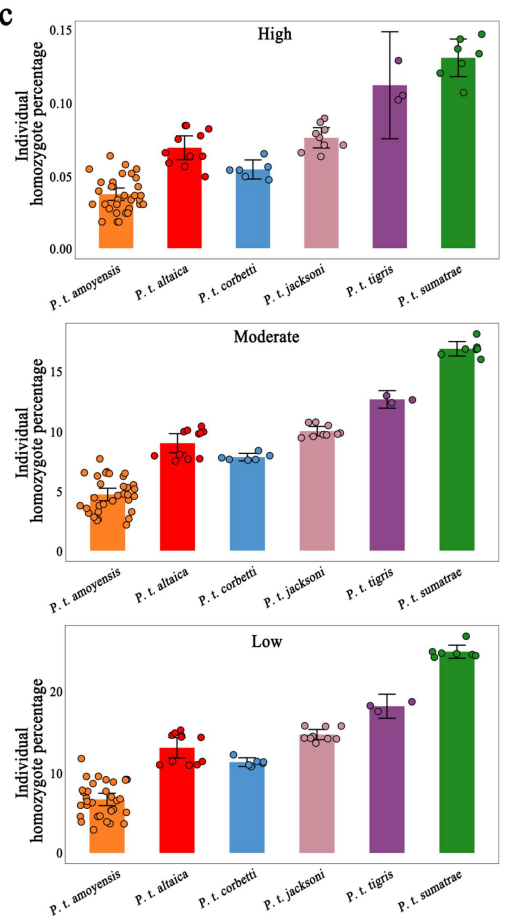

Fig. 4 | Comparison of deleterious mutations among different tiger subspecies. (a)

688 Percentage of nearly neutral (low), mildly (moderate), and highly deleterious (high impact)

689 mutations in different tiger subspecies. (b) Site frequency spectra for different impact

690 mutations in the South China tigers. Alternative allele counts $\geq 20$ are displayed as mean

691 counts per interval. (c) Individual homozygote percentage per impact category and per tiger

692 subspecies. 


\section{Supplementary Files}

This is a list of supplementary files associated with this preprint. Click to download.

- SupplementTable.pdf

- Supplementarydata.pdf 